\title{
A case report and summary of thrombocytopenia caused by coronavirus disease 2019
}

\section{Yibing Zhao}

Siping Infectious diseases Hospital, Siping, Jilin Province

\section{$\mathrm{Na} \mathrm{Du}$}

First Hospital of Jilin University

\section{Wei Li}

Siping Infectious diseases Hospital, Siping, Jilin Province

\section{Dongmei Xiang}

Siping Infectious diseases Hospital, Siping, Jilin Province

\section{Guodong Zhou}

Siping Infectious diseases Hospital, Siping, Jilin Province

\section{Ruixu Li}

Siping Infectious diseases Hospital, Siping, Jilin Province

\section{Panyang Xu}

First Hospital of Jilin University

Jiancheng Xu ( $\nabla$ xjc@jlu.edu.cn )

First Hospital of Jilin University https://orcid.org/0000-0001-8796-271X

\section{Case Report}

Keywords: Severe acute respiratory syndrome coronavirus 2,coronavirus disease 2019, thrombocytopenia, platelet

Posted Date: April 3rd, 2020

DOI: https://doi.org/10.21203/rs.3.rs-20569/v1

License: (c) (i) This work is licensed under a Creative Commons Attribution 4.0 International License. Read Full License 


\section{Abstract}

Background: Coronavirus disease 2019 has become a major problem for the world. We reported a representative case with thrombocytopenia.

Case report: A 44-year-old female patient sought medical attention for fatigue and cough of 1 day duration. Thirteen days before disease onset, the patient had returned from Hubei province. Her body temperature on admission was $38.6^{\circ} \mathrm{C}$. Blood routine test showed a white blood cell count of $4.0 \times 10^{9} / \mathrm{L}$, lymphocyte count of $1 \times 10^{9} / \mathrm{L}$, platelet count of $118 \times 10^{9} / \mathrm{L}$, and C-reactive protein of $6.0 \mathrm{mg} / \mathrm{L}$. Lung computed tomography (CT) scan showed: large patches of ground-glass opacities at the posterior segment of the right upper lung lobe and bilaterally at the basal segment of the lower lung lobes. Her throat swabs tested positive for novel coronavirus nucleic acid. After antiviral and symptomatic treatment were given, on day 5 of hospitalization, her symptoms of coughing and chest pain worsened after coughing, and she also developed hemoptysis. Repeated lung CT scans showed that the lesion area had increased. The lymphocyte count had decreased to $0.55 \times 10^{9} / \mathrm{L}$ and the platelet count had decreased to $9 \times 10^{9} / \mathrm{L}$ on day 6 . After a series of multimodal therapies were carried out, the patient's platelet and lymphocyte count gradually increased to normal.

Conclusion: We reported a COVID-2019 case with significant thrombocytopenia, which was more significantly decreased than in normal viral infections. However, the mechanism of thrombocytopenia is unknown and requires further research.

\section{Background}

Coronavirus disease 2019 (COVID-19) is a $\beta$-coronavirus infection that was caused by severe acute respiratory syndrome coronavirus-2 (SARS-CoV-2) and started to spread in Hubei province in China in December 2019. Currently, the source of infection is considered to be mainly patients with COVID-19, but asymptomatic patients and infected patients during the incubation period can also become sources of infection. The incubation period ranges from 1 to 14 days and mostly lasts for 3-7 days. At present, the "Diagnostic and treatment guideline for SARS-CoV2" (Version 7) released by the People's Republic of China National Health Committee mentions that COVID-19 mainly presents as fever, sore throat, coughing, diarrhea, and fatigue, whereas severe cases will develop dyspnea. During the early phase of COVID-19 onset, laboratory tests results will show normal or reduced white blood cell count, and reduced lymphocyte count; a few patients will have thrombocytopenia[1]. Lung computed tomography (CT) scans will show multiple, diffuse, and pale ground-glass opacities at the subpleural lateral belts in both lungs. Although cases of thrombocytopenia were reported in patients with COVID-19[1], no cases of severe thrombocytopenia were reported. In this paper, we report a patient with COVID-19 who had significant thrombocytopenia to examine the cause of the changes in platelet count and provide a theoretical basis for clinical treatment.

\section{Case Presentation:}

On January 30, 2020, a 44-year-old Chinese woman was admitted to a local infectious disease hospital for fatigue, cough, and expectoration of white sputum. Because the patient had returned to Siping in Jilin province from Wuhan (origin of COVID-19) on January 17, she underwent lung CT examinations that showed large patches of groundglass opacities at the posterior segment of the right upper lung lobe and the basal segment of the lower lung lobes bilaterally (Figure 1). 
Her throat swabs were positive for COVID-19 nucleic acid, and thus a definitive diagnosis of COVID-19 was obtained. After admission, her body temperature was $38.6^{\circ} \mathrm{C}$, pulse was $100 \mathrm{bpm}$, respiratory rate was 18 breaths/min, and blood pressure was $120 / 88 \mathrm{mmHg}$; physical examination did not show any apparent abnormalities. From January 30 to February 3, the patient's body temperature fluctuated between $37.4^{\circ} \mathrm{C}$ and $38.4^{\circ} \mathrm{C}$, and her blood oxygen saturation was between $98 \%$ and $99 \%$ with oxygen therapy. On the morning of day 5 of admission, her symptoms of coughing and productive cough worsened; traces of blood were present in the sputum, and chest pain was noted. Symptoms and Maximum Body Temperatures according to day of illness and day of hospitalization were shown in Figure 2. Compared with her previous state, her mental status, diet, and sleep were poor. Her other vital signs were essentially stable. Tests showed that on the day of admission, her white blood cell count and lymphocyte count were in reference intervals, her platelet count was slightly decreased $(118 \times 109 / \mathrm{L})$, and her lactate dehydrogenase level was increased. Table 1 shows the laboratory test results during this period. From February 1 to 4, the white blood cell count and platelet count continuously decreased. With confirmation of no blood agglutination in tubes, the blood routine tests were determined twice to determinate accurately. The patient had no history of hematologic malignancies or infectious diseases. After infusion of platelets and human immunoglobulin, laboratory tests were carried out on February 6 . The test results showed that the white blood cell count and the platelet count had increased compared with the previous results. From February 8 , the platelet count gradually returned to normal. During the hospitalization, the main treatment was Abidol oral (200mg/day, q8, and course of 10 days) and intravenous injection of methylprednisolone (80mg/day, q12, first 3 days; 40mg/day, q12, second 3 days). The patient was discharged on February 17 and the laboratory tests and lung CT examinations showed normal on February 26.

\section{Discussion And Conclusions:}

During admission, the patient's platelet count was within the reference interval and no abnormalities were found during symptomatic treatment over the inpatient observation period. Subsequently, her platelet count began decreasing on February 1 and decreased to 9×109/L on February 4. The cause of the thrombocytopenia was considered to be the SARS-CoV-2 but the effect or mechanism is unknown. A study showed that the severe acute respiratory syndrome (SARS) coronavirus can directly infect hematopoietic stem cells or blood cells through CD13 and induce myelosuppression and apoptosis, resulting in decreased primary platelet synthesis[3]. COVID-19, SARS, and MERS are all caused by $\beta$-coronaviruses and thrombocytopenia in these conditions is similar to that caused by other viral infections. Therefore, we speculate that SARS-CoV-2 may suppress hematopoiesis in the bone marrow through certain receptors to result in severe thrombocytopenia[3].

Excessive platelet destruction caused by immune factors is also one of the causes of thrombocytopenia, of which viral infection is the main trigger. According to reports, thrombocytopenia is present in various viral infections[4], and it may be caused by immunological mechanisms or the direct effects of viruses on megakaryocytes or platelets. Viral infection may result in increased autoimmune antibodies or immune complexes[5]which cause coated platelets and megakaryocytes to be considered as targets for destruction[4]. In this patient, the possibility of excessive platelet destruction due to SARS-CoV-2 or immune damage cannot be excluded. Secondary reactive thrombocytosis is common in patients with SARS and MERS $[4,6,7]$, and the mortality outcomes of these patients can be predicted from thrombocytopenia results[5].

Evidence shows that severe lung damage in patients with SARS increases platelet consumption and causes thrombocytopenia. In this patient, the lung CT scans showed large patches of ground-glass opacities, which is consistent with the chest images of COVID-19 that have been published, indicating that this patient had severe lung 
damage. It is known that the lungs may be sites in which mature megakaryocytes release platelets and that damaged lung tissues and cells causes platelet activation and aggregation to form thrombi. The formation of microthrombi increases platelet consumption. We speculate that thrombocytopenia due to damaged lungs in the patients with COVID-19 may be an alternative mechanism by which this virus induces hematologic changes[6]. Other causes resulting in thrombocytopenia include abnormal platelet distribution, such as hypersplenism, which has not been reported yet in patients with COVID-19.

The requirements of "Diagnosis and treatment protocol for novel coronavirus pneumonia" (interim edition) did not mention changes in platelet count. We found that the platelet count of this patient decreased drastically before gradually recovering to normal levels, which parallels the course of the lymphocyte changes. Conducting further studies to evaluate the mechanisms resulting in hematological changes to examine various pathogeneses and exclude other factors will help us better understand this disease. This case study showed that severe thrombocytopenia at early disease onset and its variation patterns provided a feasible basis for clinical treatment regimens for patients with COVID-19. Close monitoring of changes in platelet counts should be carried out so that potential risk factors can be discovered early. Further studies on the mechanism by which COVID-19 causes thrombocytopenia are needed.

\section{Abbreviations:}

CT, computed tomography; COVID-2019, Coronavirus disease 2019, SARS-CoV-2, Severe acute respiratory syndrome coronavirus 2 , SARS, severe acute respiratory syndrome

\section{Note:}

At present, the information of this patient is used in this study, but not in other studies.

\section{Declarations:}

Acknowledgments: We would like to thank for the participant and her families.

Authors' contributions: Conception and design of the study: Dr JC. X. and Dr YB. Zh. Investigation and statistical analysis: DM. X. and L. W. Acquisition of data: RX. L. and GD. Zh. Drafting of the manuscript: N. D. and PY. X. Revision and critical review of the manuscript: Dr JC. X. All authors reviewed and edited the manuscript and approved the final version of the manuscript.

Funding: This work was supported by grants s from Jilin Science and Technology Development Program (no.20170623092TC-09, to Dr. Jiancheng Xu; no.20190304110YY to Dr. Jiancheng Xu), The First Hospital Translational Funding for Scientific \&Technological Achievements (no.JDYYZH-1902002 to Dr. Jiancheng Xu). The funding bodies do not have any role in the design of the study and collection, analysis, and interpretation of data and in writing the manuscript.

Availability of data and materials: The datasets used and/or analysed during the current study are available from the corresponding author on reasonable request.

Ethical approval and consent to participate: This experiment is approved and reviewed by the institutional ethics committee of Siping Infectious diseases Hospital, reference number 2020-001. Written informed consent was 
obtained from the patient for the publication of this case report and the accompanying images. A copy of the written consent is available for review.

Consent for publication: Written informed consent was obtained from the patient for the publication of this case report and the accompanying images. A copy of the written consent is available for review.

Competing interests: The authors declare that they have no competing interests.

Authors' details: 1 Siping Infectious diseases Hospital, Siping, Jilin Province, 136000, China; 2 Department of Infectious Diseases, First Hospital of Jilin University, Changchun, Jilin Province, 130021, China; 3 Department of Laboratory Medicine, First Hospital of Jilin University, Changchun, Jilin Province, 130021, China.

\section{References}

[1] CHEN N, ZHOU M, DONG X, et al. Epidemiological and clinical characteristics of 99 cases of 2019 novel coronavirus pneumonia in Wuhan, China: a descriptive study [J]. Lancet, 2020,

[2] ARABI Y M, ARIFI A A, BALKHY H H, et al. Clinical course and outcomes of critically ill patients with Middle East respiratory syndrome coronavirus infection [J]. Ann Intern Med, 2014, 160(6): 389-97.

[3] YANG M, LI C K, LI K, et al. Hematological findings in SARS patients and possible mechanisms (review) [J]. Int J Mol Med, 2004, 14(2): 311-5.

[4] WONG R S, WU A, TO K F, et al. Haematological manifestations in patients with severe acute respiratory syndrome: retrospective analysis [J]. BMJ, 2003, 326(7403): 1358-62.

[5] YANG M, NG M H, LI C K. Thrombocytopenia in patients with severe acute respiratory syndrome (review) [J]. Hematology, 2005, 10(2): 101-5.

[6] MULLER M P, RICHARDSON S E, MCGEER A, et al. Early diagnosis of SARS: lessons from the Toronto SARS outbreak [J]. Eur J Clin Microbiol Infect Dis, 2006, 25(4): 230-7.

[7] YIN Y, WUNDERINK R G. MERS, SARS and other coronaviruses as causes of pneumonia [J]. Respirology, 2018, 23(2): 130-7.

\section{Table 1}

\section{Figures}


Table 1. Clinical Laboratory Results

\begin{tabular}{|c|c|c|c|c|c|c|c|c|c|c|c|c|c|c|}
\hline Measure & $\begin{array}{l}\text { Reference } \\
\text { interval }\end{array}$ & Jan.30 & Feb.1 & Feb.4 & Feb.5 & Feb.6 & Feb.7 & Feb.8 & Feb.9 & Feb.10 & Feb.11 & Feb.13 & Feb.17 & Feb.26 \\
\hline White blood cell $\left(10^{9} / \mathrm{L}\right)$ & $4.0-10.0$ & 4.0 & $3.6 \downarrow$ & $2.4 \downarrow$ & $3.3 \downarrow$ & 5.3 & 5.3 & 7.3 & 7.4 & 8.5 & 7.1 & 9.0 & 6.1 & 6.0 \\
\hline Red blood cells $\left(10^{12} / \mathrm{L}\right)$ & $3.5-5.5$ & 4.4 & 4.2 & 4.3 & 4.0 & 4.1 & 4.1 & 4.3 & 4.0 & 3.8 & 3.9 & 3.6 & 3.8 & 3.9 \\
\hline Hemoglobin $(\mathrm{g} / \mathrm{L})$ & $110-160$ & 144 & 135 & 138 & 125 & 131 & 133 & 137 & 128 & 124 & 123 & 116 & 125 & 127 \\
\hline Hematocrit (\%) & $\begin{array}{l}0.370- \\
0.500\end{array}$ & 0.416 & 0.397 & 0.400 & 0.377 & 0.384 & 0.384 & 0.398 & 0.372 & $0.358 \downarrow$ & $0.360 \downarrow$ & $0.340 \downarrow$ & $0.369 \downarrow$ & 0.378 \\
\hline Neutrophils $\left(10^{9} / \mathrm{L}\right)$ & $2.0-7.0$ & 2.5 & 2.3 & $1.7 \downarrow$ & 2.4 & 4.2 & 4.0 & 5.4 & 5.6 & 6.6 & 3.7 & 6.3 & 4.2 & 4.3 \\
\hline Lymphocytes $\left(10^{9} / \mathrm{L}\right)$ & $0.8-4.0$ & 1.0 & 0.9 & $0.6 \downarrow$ & $0.7 \downarrow$ & 0.8 & 1.0 & 1.2 & 1.2 & 1.3 & 2.7 & 1.8 & 1.1 & 1.1 \\
\hline Platelets count $\left(10^{9} / \mathrm{L}\right)$ & $100-300$ & 118 & $38 \downarrow$ & $9 \downarrow$ & $27 \downarrow$ & $71 \downarrow$ & $77 \downarrow$ & 119 & 143 & 176 & 153 & 140 & 100 & 126 \\
\hline $\begin{array}{l}\text { High-sensitivity } \quad \text { C- } \\
\text { reactive protein }(\mathrm{mg} / \mathrm{L})\end{array}$ & $0-10.0$ & 6.0 & 8.8 & 6.5 & 2.4 & 0.4 & 0.5 & 0.7 & 0.5 & 0.5 & 0.5 & 1.7 & - & - \\
\hline Creatine kinase (U/L) & $26-174$ & 49 & 59 & - & - & - & 26 & - & $20 \downarrow$ & - & $24 \downarrow$ & 30 & $25 \downarrow$ & - \\
\hline $\begin{array}{l}\text { Lactate dehydrogenase } \\
(\mathrm{U} / \mathrm{L})\end{array}$ & $109-245$ & $260 \uparrow$ & 245 & - & - & - & $257 \uparrow$ & - & 226 & - & 178 & 167 & 190 & - \\
\hline $\begin{array}{l}\text { Aspartate } \\
\text { aminotransferase (U/L) }\end{array}$ & $15-40$ & 29 & 28 & - & - & - & 31 & - & 27 & - & 22 & 22 & 23 & 20 \\
\hline $\begin{array}{l}\text { Alanine } \\
\text { aminotransferase (U/L) }\end{array}$ & $9-50$ & 28 & 20 & - & - & - & $64 \uparrow$ & - & $72 \uparrow$ & - & $72 \uparrow$ & $59 \uparrow$ & 48 & 30 \\
\hline $\begin{array}{l}\mathcal{\gamma} \text {-glutamyl } \\
\text { transpeptidase }(\mathrm{U} / \mathrm{L})\end{array}$ & $10-60$ & 28 & 21 & - & - & - & 38 & - & 36 & - & 33 & 36 & 34 & 33 \\
\hline $\begin{array}{l}\text { Alkaline phosphatase } \\
(\mathrm{U} / \mathrm{L})\end{array}$ & $45-125$ & $30 \downarrow$ & $25 \downarrow$ & - & - & - & $34 \downarrow$ & - & $29 \downarrow$ & - & $31 \downarrow$ & $37 \downarrow$ & $30 \downarrow$ & $42 \downarrow$ \\
\hline Total protein $\mathrm{g} / \mathrm{L}$ & $60-83$ & 72.5 & 61.0 & - & - & - & 70.5 & - & 71.8 & - & 62.0 & 60.2 & 64.2 & 66.5 \\
\hline Albumin (g/L) & $40-55$ & 42.1 & $36.3 \downarrow$ & - & - & - & $34.5 \downarrow$ & - & $30.7 \downarrow$ & - & $29.0 \downarrow$ & $30.2 \downarrow$ & $34.1 \downarrow$ & $38.1 \downarrow$ \\
\hline Total bilirubin $(\mu \mathrm{mol} / \mathrm{L})$ & $3.4-17.1$ & 13.6 & 15.7 & - & - & - & $26.3 \uparrow$ & - & $32.2 \uparrow$ & - & $18.1 \uparrow$ & 13.7 & 11.9 & 17.0 \\
\hline Direct bilirubin $(\mu \mathrm{mol} / \mathrm{L})$ & $0-3.4$ & 3.4 & $4.4 \uparrow$ & - & - & - & $7.8 \uparrow$ & - & $9.1 \uparrow$ & - & $6.2 \uparrow$ & $5.4 \uparrow$ & $5.7 \uparrow$ & $4.8 \uparrow$ \\
\hline Urea (mmol/L) & $2.9-8.2$ & 3.0 & $1.9 \downarrow$ & - & - & - & 4.7 & - & $8.5 \uparrow$ & - & 5.6 & 4.3 & 3.1 & 4.2 \\
\hline Creatinine $(\mu \mathrm{mol} / \mathrm{L})$ & 54-109 & 60 & 63 & - & - & - & 56 & - & 73 & - & 63 & 64 & 62 & 62 \\
\hline $\begin{array}{l}\text { Carbondioxide combining } \\
\text { power (mmol/L) }\end{array}$ & $23.0-29.0$ & $22.4 \downarrow$ & $21.5 \downarrow$ & - & - & - & 25.2 & - & 28.3 & - & 27.0 & 28.6 & 27.9 & 26.7 \\
\hline Glucose (mmol/L) & $3.90-6.10$ & 5.39 & 5.34 & - & - & - & 5.78 & - & $2.91 \downarrow$ & - & 4.06 & $3.49 \downarrow$ & 5.30 & 4.23 \\
\hline Potassium (mmol/L) & $3.5-5.3$ & $3.2 \downarrow$ & 3.6 & - & - & - & 3.8 & - & 3.5 & - & 3.5 & $3.3 \downarrow$ & 3.7 & 3.8 \\
\hline Sodium (mmol/L) & $137-147$ & 137 & 141 & - & - & - & 146 & - & 140 & - & 140 & $129 \downarrow$ & $136 \downarrow$ & 139 \\
\hline Chloride (mmol/L) & $99-110$ & $94 \downarrow$ & 101 & - & - & - & 101 & - & 103 & - & 101 & 102 & 105 & 101 \\
\hline
\end{tabular}

Note: $\uparrow$ for higher than the upper limit of reference interval; $\downarrow$ for below than the lower limit of reference interval 

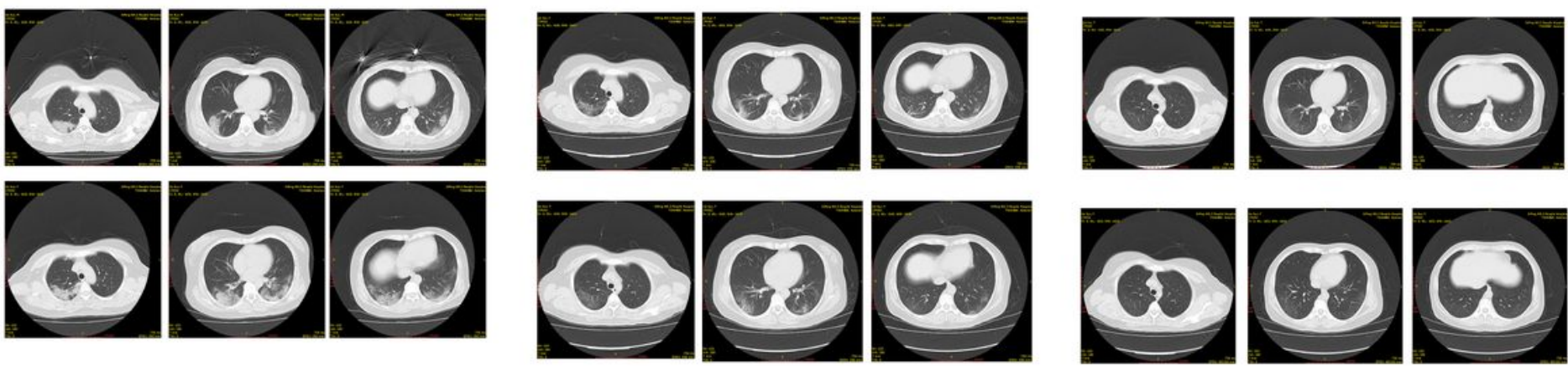

Figure 1

The first image Figure 1 is a CT image of the patient on January 30 and February 5, respectively Figure 1 shows the CT images of the patients on February 9 and February 13, respectively $\triangle 1$ 1 Figure 1 is the CT image of the patient on February 26 and March 3, respectively

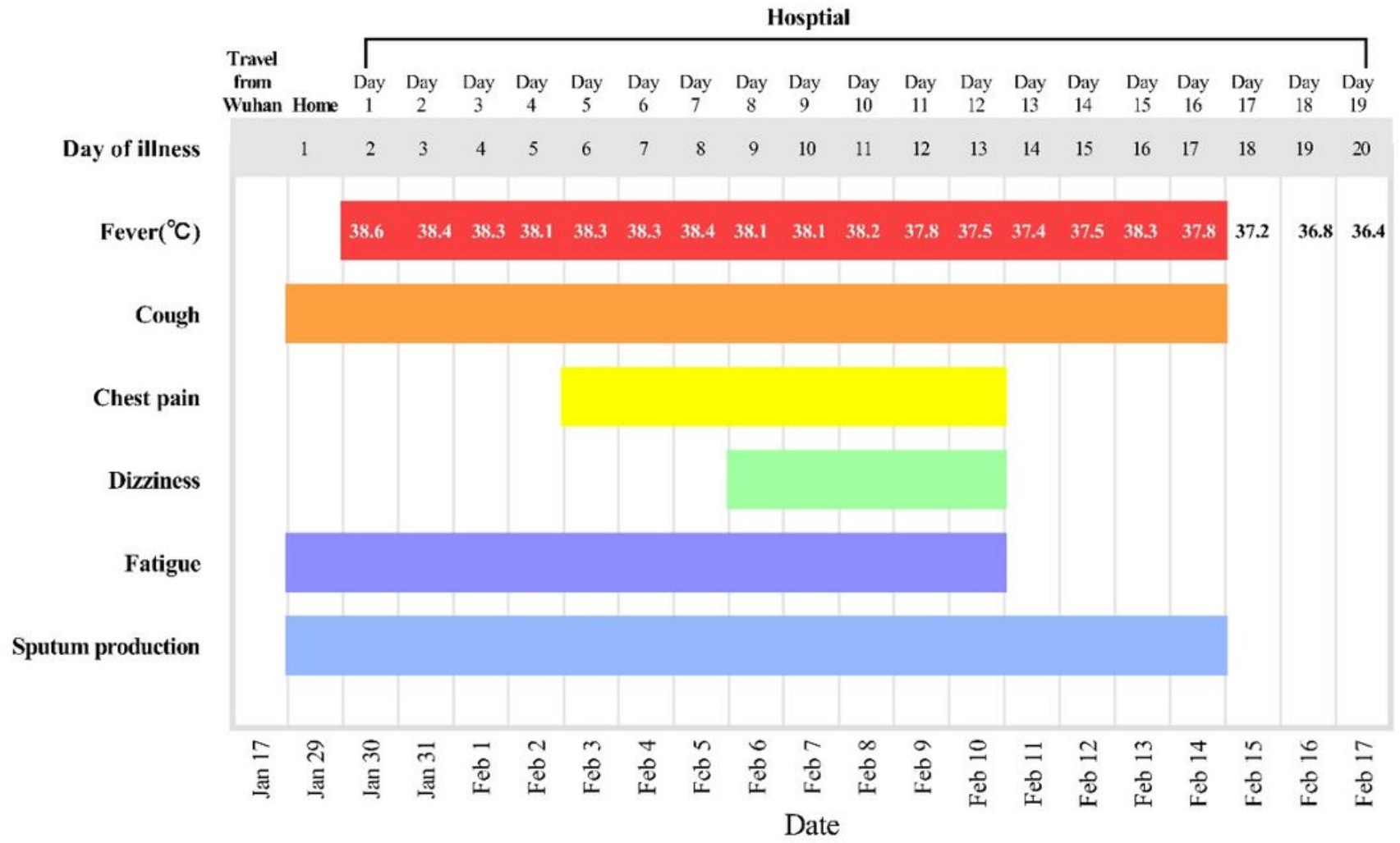

Figure 2

Symptoms and Maximum Body Temperatures according to day of illness and day of hospitalization were shown in Figure 2. 